\title{
Les matérialités discursives et les pratiques citationnelles du discours rapporté numérique sur Instagram
}

\author{
Grégoire Lacaze ${ }^{1, *}$ \\ ${ }^{1}$ Aix-Marseille Université, LERMA, Aix-en-Provence, France
}

\begin{abstract}
Résumé. La présente étude s'attache à décrire les matérialités discursives et les pratiques citationnelles à l'œuvre sur le RSN Instagram. La plurisémioticité des publications numériques nécessite une inflexion paradigmatique dans l'analyse de ces discours sous l'effet de la délinéarisation et de l'hypertextualité. Cette recherche analyse comment les publications et les stories postées sur Instagram renouvellent les processus de citation et de partage, grâce à l'exploitation des fonctionnalités technologiques de la plateforme numérique.
\end{abstract}

\begin{abstract}
Quoting and using digital reported speech on Instagram. This study aims to describe the quoting strategies implemented on the social media Instagram. The plurisemioticity of digital posts requires to adopt a new methodological approach to discourse analysis thanks to delinearisation and hypertextuality. This research focuses on the way posts and stories published on Instagram alter the processes of quoting and sharing speeches, due to the various technological functions offered by the digital platform.
\end{abstract}

\section{Introduction}

Les discours numériques produits sur les réseaux socionumériques (RSN) nécessitent d'adopter une approche méthodologique d'analyse ayant subi une inflexion notable par rapport à l'analyse de discours plus traditionnellement mise en œuvre pour l'étude de corpus textuels de fiction ou de presse.

La nature même du support numérique sur lequel ces discours prennent naissance amène à prendre en compte la double composante qui les caractérise : une composante langagière (une production verbale effective) et une composante technologique (informatique, en l'occurrence).

Les linguistes, analystes du discours et chercheurs en sciences de l'information et de la communication se sont penchés depuis une vingtaine d'années sur ces discours numériques pour identifier, analyser et classer ces productions verbales caractérisées par une pluriosémioticité (ou polysémioticité) intrinsèque.

\footnotetext{
* gregoire.lacaze@univ-amu.fr
} 
La présente recherche s'inscrit dans le sillage des travaux récents en linguistique et analyse du discours qui décrivent ces discours de nature composite (Angé, 2015, Basso Fossali et al, 2019, Bigey et Simon, 2018, Chevet et Garmon, 2019, Constantin de Chanay et Rosier, 2016, Grossmann et Rosier, 2018, Jackiewicz, 2016, Longhi et Weber, 2018, López Muñoz, 2017, Paveau, 2017, Petitjean et al., 2017, Reboul-Touré, 2016, Saemmer, 2015, notamment)

Elle analyse les matérialités discursives d'Instagram à partir de l'étude d'occurrences de discours rapporté produites sur ce RSN sur le compte officiel du président français Emmanuel Macron avec une mise en perspective avec les pratiques citationnelles adoptées par le président américain Donald Trump ${ }^{1}$ sur Instagram.

\section{Les singularités des discours numériques sur les RSN}

\subsection{Vers une inflexion paradigmatique de l'analyse du discours}

Les discours numériques sont des discours produits et circulant sur Internet comme les sites web et les plateformes numériques des RSN. Il s'agit ici de prendre en compte les discours dont la matérialité même s'inscrit dans un écosystème numérique qui permet à ces contributions d'être repérées les unes par rapport aux autres par des identifiants uniques. Nous soulignons ici la nature réticulaire des discours numériques, au sens étymologique du terme, qui évoque le maillage existant entre ces différentes contributions numériques qui constituent un réseau.

Comme le soulignent C. Develotte et M.-A. Paveau (2017:201) : « les discours natifs de l'internet, et du web en particulier, présentent des traits proprement technolangagiers et technodiscursifs, coproduits par les scripteurs usagers et les affordances du système ». Les deux linguistes militent pour une approche méthodologique spécifique pour analyser les discours numériques: "Une analyse du discours numérique fondée sur une approche linguistique écologique, c'est-à-dire intégrant les dimensions technologiques des univers numériques, peut selon nous rendre compte de la complexité des énoncés natifs en ligne dans leurs contextes sociotechniques » (Develotte et Paveau, $2017: 201$ ).

La nature composite de toute production langagière numérique associant une composante verbale et une composante technologique est intrinsèque à toute publication sur les RSN : «En ligne, les données langagières ne sont pas constituées de matières purement langagières, mais sont composites, métissées de non-langagier de nature technique » (Develotte et Paveau, $2017: 205$ ).

F. Grossman et L. Rosier (2018:44) défendent plutôt une perspective «continuiste » pour analyser ces discours numériques, qui requièrent à la fois l'expertise (plus) classique d'analyse des discours produits sur des supports textuels tout en intégrant les fonctionnalités technologiques nouvelles que les plateformes numériques offrent aux contributeurs: "Pour nous, les rapports entre discours rapporté et hypertextualité s'inscrivent dans une approche continuiste des pratiques numériques qui reconfigurent des pratiques et des genres anciens $\gg$.

\subsection{La délinéarisation et l'hypertextualisation}

Les discours nativement numériques que l'on rencontre sur les RSN sont marqués par leur plurisémioticité. Ils reposent sur l'intégration de fragments de discours numériques plus ou moins imbriqués et sur l'interactivité revendiquée et recherchée par les utilisateurs de ces réseaux.

M.-A. Paveau (2016: 41) souligne un trait commun à tous les discours numériques : ils présentent une «"délinéarisation technodiscursive" caractérisée par l'élaboration d'un lien 
avec un autre fil de discours ", ce qui permet de relier un discours numérique donné à un ou plusieurs autres discours numériques. Ainsi, un discours numérique appartient, par essence, à une " galaxie » de discours potentiellement connectés les uns aux autres par ce que nous appelons un « effet de relationnalité ».

L'appartenance à un écosystème assurant la mise en relation de toutes les productions numériques propres à un RSN repose sur la réticularité :

l'ensemble des éléments langagiers et discursifs qui sont produits en ligne sont dotés de ce caractère composite du fait même de la nature de leur écosystème de production : la réticularité d'internet construite par les algorithmes implique en effet que tous les énoncés en ligne constituent des liens; la relationalité est un trait structurant des discours numériques natifs. (Develotte et Paveau, 2017 : 205-206)

Cette propension à l'interconnexion d'un discours numérique avec d'autres discours numériques repose sur l'exploitation du principe d' « hypertextualité » (Saemmer 2015).

\subsection{L'incomplétude ontologique des discours numériques des RSN}

Les discours numériques sont hébergés par des plateformes « ouvertes» sur lesquelles les contributeurs peuvent réagir en commentant et en postant des publications sans limite de temps et en s'affranchissant des frontières géographiques : n'importe quel internaute peut commenter, «liker» une publication qui a été créée par un tiers habitant un autre pays ou une région géographiquement très éloignée de la sienne.

Chaque plateforme de RSN se présente donc comme un système ouvert caractérisé par son incomplétude ontologique. Toutes ces plateformes sont enrichies en permanence de nouvelles entrées et de commentaires en fonction des actions des utilisateurs. À l'intérieur d'un système ouvert, chaque contribution est elle-même ouverte aux actions des internautes :

l'inachèvement, ou, pour utiliser un terme sans préfixe négatif, l'ouverture du texte numérique, est typique, sur le plan du genre et du dispositif énonciatif, des blogs, qui sont des espaces textuels conversationnels où les commentateurs augmentent les billets des blogueurs et deviennent par la même auteurs. (Paveau, $2015: 346$ )

\subsection{L'unicité du corpus numérique constitué}

L'incomplétude ontologique d'un RSN fait que l'acquisition des données pour constituer un corpus offre un instantané à un moment donné précis (horodaté) de l'état des données stockées sur une plateforme numérique, ce corpus évoluant en permanence au gré des interactions des utilisateurs. Nous soulignons ici le statut unique d'un corpus numérique : la collecte de publications numériques à un instant donné pour construire un corpus en vue d'une analyse fige ces données tandis que les publications sur la plateforme numérique d'un RSN continuent d'évoluer sans cesse.

Comme le soulignent F. Sitri et C. Barats (2017 41), le corpus est «construit en fonction des questions et des hypothèses de recherche, voire même en fonction de la conception que l'on a de l'analyse du discours, ou encore des outils ou des catégories que l'on utilise ».

Il faut donc toujours avoir présent à l'esprit la relativité d'un corpus de publications numériques, la nature ouverte du médium entraînant son instabilité permanente. C. Barats, J.-M. Leblanc et P. Fiala (2017 : 112) aboutissent à un constat assez similaire :

Ainsi, tout corpus web, stabilisé et documenté à un moment donné, ne peut être considéré comme un échantillon au sens strict du terme, mais 
doit être regardé plutôt comme un état de données provisoire, évolutif, prélevé au sein d'une archive vivante, gardant une marge d'incertitude non négligeable.

Comme toute publication numérique peut être enrichie à tout instant de commentaires d'internautes, une publication n'est pas figée et son contenu même ainsi que ses métadonnées sont potentiellement modifiables en permanence :

\begin{abstract}
Les énoncés numériques natifs sont doublement innombrables : d'une part, contrairement aux énoncés hors ligne (ouvrages, journaux, échanges oraux), ils ne connaissent pas de clôture, toujours transformables, par délinéarisation ou augmentation notamment; d'autre part, internet étant un univers relationnel, et le web social permettant la fonction de partage, tout énoncé est susceptible d'être remis en circulation dans d'autres contextes que son contexte d'origine, et d'y être de nouveau délinéarisé ou augmenté. (Develotte et Paveau, $2017: 207)$
\end{abstract}

La spécificité d'un corpus numérique rassemblant des publications d'un RSN est son unicité : à peine est-il constitué qu'il est déjà quasiment impossible pour un autre chercheur de reconstituer un corpus contenant le même jeu de données.

Le corpus numérique constitué à dessein pour cette recherche a été figé au 6 octobre 2019 : il rassemble 434 publications (compte@emmanuelmacron) et 5055 publications (compte@realdonaldtrump) ainsi que des captures d'écran correspondant aux publications éphémères que représentent les « stories».

La matérialité du corpus ainsi constitué à partir des stories Instagram présente des spécificités qui seront envisagées, notamment la nature plurisémiotique de ces publications pouvant associer des images animées et du son, ce qui implique nécessairement des choix pour la présentation de ces publications dans un article de recherche.

\title{
3 Les publications postées sur Instagram
}

Instagram, le RSN actuellement le plus populaire chez les jeunes Américains et Européens, a été créé en 2010. Il a été racheté ensuite par Facebook en 2012. Plus d'un milliard d'utilisateurs utilisent cette plateforme chaque mois, ce qui montre son succès phénoménal.

\subsection{Les spécificités fonctionnelles d'Instagram}

Ce RSN a été créé à l'origine comme une plateforme d'échange de photos mais il a très vite accueilli des vidéos de courte durée. La fonction «Stories » a été ajoutée en août 2016 au RSN pour concurrencer le RSN Snapchat, qui doit son grand succès à cette fonctionnalité très populaire et appréciée de ses utilisateurs. Elle contient des contenus éphémères (d'une durée limitée à 24 heures) qui s'affichent dans le profil du compte et qui disparaissent le jour suivant. Il est également possible pour le gestionnaire d'un compte de créer différents dossiers de stories visibles sous le profil d'un compte (stories «à la une »), celles-ci n'étant pas à durée limitée. Elles peuvent donc continuer à être visionnées des jours, des semaines, voire des mois ou des années après leur mise en ligne.

À l'instar de Twitter, il est possible de partager aussi bien des photos que des vidéos sur Instagram. Toutes les publications sont présentées de manière synthétique sous la forme d'images carrées présentant soit une photo soit une capture d'écran de vidéo ; ces images sont alignées sur une grille, avec une présentation assez semblable à celle du RSN Flickr ${ }^{3}$ spécialisé dans l'hébergement de galeries photographiques. 


\subsection{L'écriture d'une publication sur Instagram}

Lors de la création d'une publication sur Instagram, il est possible de publier une photo ou une vidéo enregistrée sur le terminal multimédia mais il est également envisageable de partager une photo ou une vidéo déjà publiée sur un autre RSN en utilisant des fonctions de partage proposées par le smartphone ou la tablette. Ainsi la circulation transmédiale (ou trans-RSN) est une caractéristique des publications numériques qui mérite d'être soulignée.

Contrairement au RSN Twitter, Instagram n'autorise pas la publication de contributions à partir du navigateur Internet d'un ordinateur. Il est nécessaire d'utiliser l'application (préalablement téléchargée) à partir d'un smartphone ou d'une tablette.

Le tableau ci-dessous offre un comparatif des fonctionnalités technologiques offertes par Twitter et Instagram :

Tableau 1. Tableau comparatif des fonctionnalités technolangagières de Twitter et d'Instagram

\begin{tabular}{|c|c|c|}
\hline & Twitter & Instagram \\
\hline Publier à partir d'un ordinateur & $\mathrm{X}$ & \\
\hline $\begin{array}{l}\text { Publier à partir d'une application } \\
\text { mobile }\end{array}$ & $\mathrm{X}$ & $\mathrm{X}$ \\
\hline Publier des « stories » & & $\mathrm{X}$ \\
\hline Contenu de base & $\begin{array}{c}\text { Chaîne de caractères } \\
\text { limitée à } 280 \text { signes } \\
\text { (contenant du texte, des } \\
\text { emojis, des hyperliens ou } \\
\text { autres technomots) } \\
\text { ou image fixe ou animée } \\
\text { ou sondage }\end{array}$ & Image fixe ou animée \\
\hline Contenus enrichis & $\begin{array}{l}\text { Combinaison des } \\
\text { contenus de base } \\
\text { ou iconotexte }\end{array}$ & $\begin{array}{l}\text { Chaîne de caractères } \\
\text { (contenant du texte, des } \\
\text { emojis, des hyperliens ou } \\
\text { autres technomots) }\end{array}$ \\
\hline
\end{tabular}

Puisqu'une publication numérique est, par nature, ouverte à la plurisémioticité, une photo ou une vidéo est accompagnée d'une zone de saisie dans laquelle peuvent cohabiter des chaînes de caractères incluant du texte et des « technomots " ${ }^{4}$ (hashtags, mention d'un compte avec@...) ainsi que des emojis. Par ailleurs, la saisie d'un hashtag conduit à la consultation et à l'actualisation en temps réel de la base de données contenant l'ensemble des hashtags de la plateforme avec des suggestions d'autres hashtags apparentés ainsi que le nombre d'occurrences d'un hashtag donné. Ces informations sont très précieuses pour l'indexation de la publication dans la base et sa « redocumentarisation $»^{5}$. Il est possible d'enrichir la base par l'ajout d'un hashtag si celui-ci n'y existe pas déjà.

La mention d'un compte tiers via l'utilisation de @ a une fonction d'interpellation et de monstration. Elle génère l'envoi d'une notification au gestionnaire du compte mentionné, ce qui contribue au rayonnement d'une publication par l'exploitation de la dimension réticulaire du RSN. L'analyse des liens et mentions croisés entre comptes souligne l'importance de la relationnalité et de la viralité.

\section{4 Études de cas sur Instagram}

La présente recherche étudie les pratiques citationnelles adoptées par le président français Emmanuel Macron dans sa communication institutionnelle sur Instagram avec une mise en perspective avec les stratégies citationnelles adoptées par le président américain 
Donald Trump sur ce même RSN. Elle analyse les deux types de contributions offertes par le RSN : envoi de publications et diffusion de « stories ».

\subsection{L'identité numérique liée à la construction d'un « ethos socionumérique »}

L'identité numérique d'un contributeur s'établit à partir des informations textuelles et graphiques qui constituent son profil (choix de l'avatar, texte de description...), comme cela a été vu dans les figures 1 et 2 . La description du profil s'appuie sur une séquence technodiscursive pouvant contenir aussi bien du texte que des emojis ou des hyperliens.

À partir de l'ensemble des publications d'un compte donné sur un RSN, s'établit ce que nous appelons un «ethos socionumérique » pour ce contributeur, ces publications reflétant des traits subjectifs : en effet, le choix des contenus sémiotiques publiés (textes, images, vidéos, emojis...) est porteur de sens et laisse affleurer la subjectivité du contributeur. Cet ethos socionumérique s'affine et évolue au cours du temps en fonction des nouvelles publications postées par un contributeur.

\subsection{Les publications postées}

La paucité des contributions sur le compte Instagram d'Emmanuel Macron met en relief la rareté de la parole présidentielle sur ce RSN. Il est intéressant de comparer les stratégies discursives très différentes adoptées par le président français et le président américain, qui utilise abondamment les réseaux sociaux avec de nombreuses contributions quotidiennes :
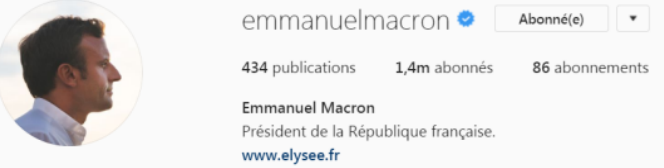

Fig. 1. Profil du compte d'Emmanuel Macron.

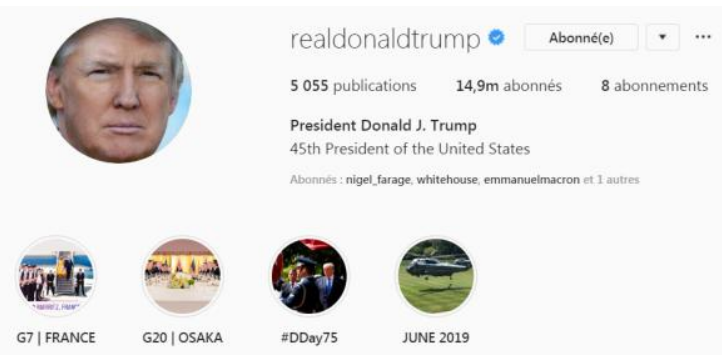

Fig. 2. Profil du compte de Donald Trump.

Le compte@emmanuelmacron compte 433 publications au 28 septembre 2019 alors que le compte@realdonaldtrump en compte 5030. Par ailleurs, le compte du président de la République française ne contient pas de stories à la une contrairement à celui de Donald Trump.

La première publication d'Emmanuel Macron remonte au 3 septembre 2016, quelques jours après qu'il a quitté son poste de ministre de l'Économie, de l'Industrie et du Numérique : 


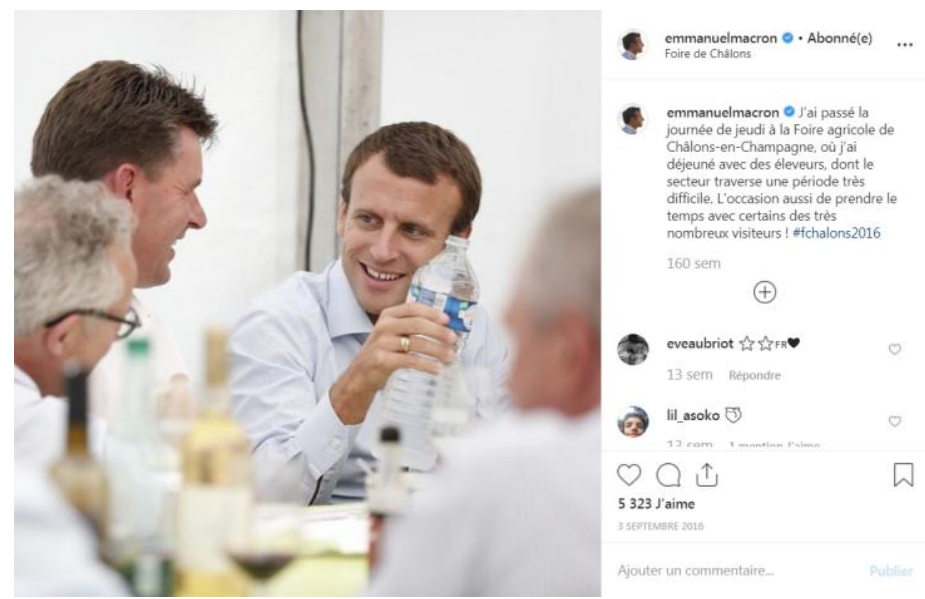

Fig. 3. Première publication sur le compte d'Emmanuel Macron.

La publication est explicitement attribuée au compte@emmanuelmacron (qui a été vérifié par la plateforme du RSN, ce qui permet d'attester la source énonciative du post). L'horodatage de la publication (le 3 septembre 2016) et la localisation géographique (la Foire de Châlons) ancrent cet acte énonciatif dans un cadre spatio-temporel. L'énonciation contient deux phrases suivies du hashtag \#fchalons2016, permettant de mettre en relation thématique cette publication avec toutes celles ayant trait à cette foire agricole. Les interactions les plus récentes sont présentées en dessous de l'énonciation d'Emmanuel Macron ainsi que le nombre de contributeurs ayant aimé cette publication. Les «technosignes ${ }^{6}$ (ou technoboutons) ainsi que la zone de saisie d'un commentaire constituent autant d'appels à l'interaction des contributeurs.

Quand une image a fait l'objet d'un traitement graphique ${ }^{7}$ (avec notamment l'ajout d'un contenu propositionnel sous la forme d'un segment textuel), il ne s'agit plus d'une simple photo mais bien d'un document iconographique enrichi, ce que M.-A. Paveau (2017 305) appelle un «technographisme»: «On appellera technographisme une production sémiotique associant texte et image dans un composite natif d'internet ».

La publication ci-dessous illustre un tel usage :

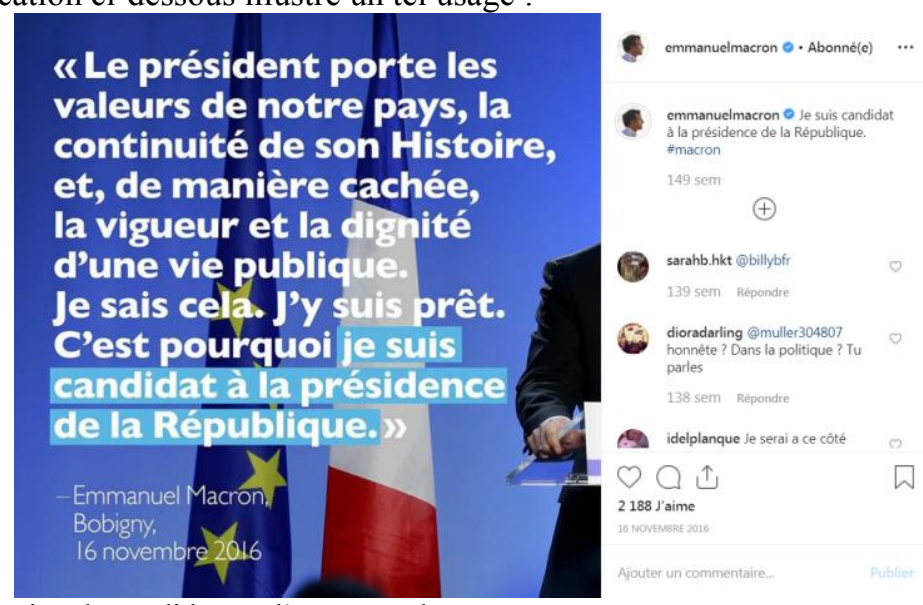

Fig. 4. Déclaration de candidature d'Emmanuel Macron.

Dans cette publication exploitant la plurisémioticité de l'illustration, le texte occupe une place en surplomb par rapport à l'image. La citation reproduite ici contient la déclaration officielle de candidature prononcée à Bobigny le 16 novembre 2016. Le texte accompagnant l'iconographie a une valeur performative : «Je suis candidat à la présidence 
de la République ». Le fragment textuel du discours du candidat a été sélectionné par son équipe de communication et la séquence d'annonce de sa candidature a été mise en surbrillance.

Deux jours avant le premier tour des élections présidentielles, le candidat a posté une citation des Misérables de Victor Hugo, qu'il commente ainsi :

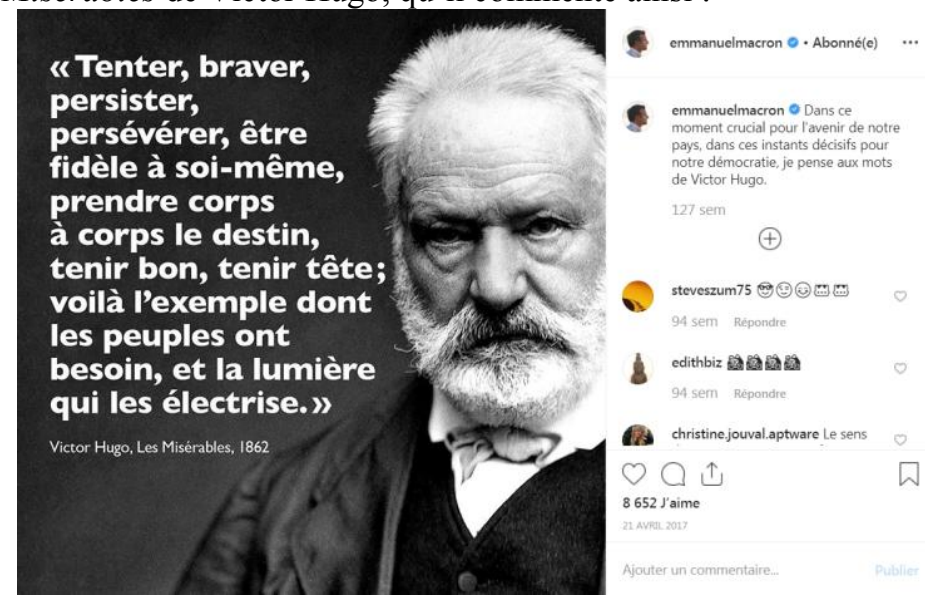

Fig. 5. Citation de Victor Hugo.

Dans ce dispositif énonciatif, l' «hyperénonciateur ${ }^{8}$ (le RSN Instagram) dit qu'Emmanuel Macron « pense aux mots de Victor Hugo ». Le candidat est donc à la fois un locuteur cité et un locuteur citant. S'instaure alors une chaîne d'énonciateurs aboutissant à la circulation de la citation hugolienne s'apparentant à " une particitation scripturaire », comme D. Maingueneau (2004: 115) appelle « les citations célèbres ».

L'auto-citation transmédiale/trans-RSN est aussi une stratégie souvent mise en œuvre par les acteurs politiques pour diffuser et faire circuler leurs "petites phrases » ${ }^{9}$ et les partager avec d'autres contributeurs. Intéressons-nous à la genèse de cette publication :

Emmanuel Macron
J'ai demandé au gouvernement de
prendre les dispositions nécessaires pour
que l'utilisation du glyphosate soit
interdite en France dès que des
alternatives auront été trouvées, et au plus
tard dans 3 ans.
\#MakeOurPlanetGreatAgain
27/11/2017 18:10

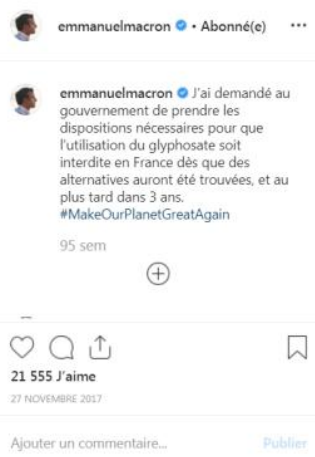

Fig. 6. Auto-citation transmédiale d'Emmanuel Macron.

La publication originelle est un tweet d'Emmanuel Macron publié le 27 novembre 2017 sur le RSN Twitter. Ce tweet contient une occurrence de discours rapporté, plus précisément une occurrence de discours indirect. Le président rapporte les instructions qu'il a données au gouvernement. Une capture d'écran de la publication sur Twitter devient une publication iconographique, iconotextuelle postée sur Instagram. Le changement de RSN s'est accompagné de la perte de l'hypertextualité : les hyperliens cliquables sur Twitter ne le sont plus dans l'image-texte publiée sur le compte Instagram. Toutefois, la zone dévolue à la saisie de chaînes de caractères dans la publication sur Instagram contient exactement le même contenu textuel enrichi que celui posté initialement sur Twitter et le hashtag 
\#MakeOurPlanetGreatAgain ${ }^{10}$ est bien activable pour une redocumentarisation sur Instagram.

Notre étude comparative des publications d'Emmanuel Macron et de Donald Trump dans leur utilisation respective de Twitter et d'Instagram montre que ces dirigeants politiques préfèrent généralement rédiger en premier une publication sur Twitter, avant que celle-ci ne soit reprise (par une simple capture d'écran, le plus souvent) dans une publication sur Instagram. Ainsi, ils utilisent le RSN Instagram comme un relais de diffusion institutionnelle de leurs tweets auprès d'une communauté élargie de contributeurs. Ils choisissent aussi d'utiliser Instagram pour diffuser du contenu inédit sous forme de stories, comme cela est étudié ensuite.

Par ailleurs, la publication reproduite ci-dessus présente une certaine redondance du contenu verbal que peuvent lire les contributeurs. Elle est aussi caractérisée par une dualité lecture/écriture: la lecture d'un document non cliquable dans sa partie gauche mais activable dans sa partie droite, l'action possible d'un contributeur pouvant ajouter un commentaire sous une forme écrite. Elle illustre les propriétés d' « écrilecture » ${ }^{11}$ associées aux publications composites générées sur les plateformes numériques.

L'auto-citation et la réénonciation cohabitent dans cette publication sur Instagram. Ces deux opérations sont fréquemment utilisées par les acteurs politiques pour améliorer la diffusion d'une information sur des RSN concurrents pour en maximiser l'audience. L'instantanéité semble prévaloir avec Twitter (publications postées en temps réel sans décalage temporel par rapport à l'actualité) alors que les publications diffusées sur Instagram semblent davantage faire l'objet d'un retravail par une équipe de communication.

La publication d'Emmanuel Macron met en avant une opération relativement simple qui est une capture d'écran. La mise en forme de la publication peut être bien plus élaborée quand l'iconotexte $^{12}$ est considérablement retravaillé (emploi de différentes polices, d'italiques, et, éventuellement, changement de couleur ou mise en surbrillance de fragments textuels) comme dans la publication suivante postée sur le compte de Donald Trump :
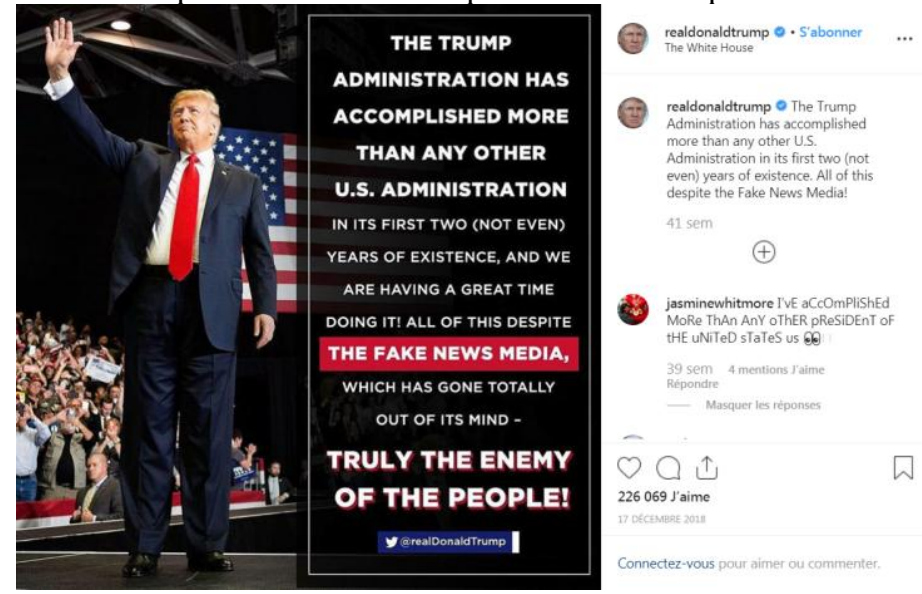

Fig. 7. Auto-citation transmédiale de Donald Trump.

L'iconotexte plurisémiotique contient une zone de texte bien délimitée par un cadre dans lequel le fragment du discours présidentiel est détaché de l'énonciation originelle publiée sur Twitter. Le logo de Twitter et la mention du compte du président américain apparaissent en dessous des paroles proférées, ce qui montre qu'Instagram republie une citation publiée au préalable sur un autre RSN. Il s'agit également d'une citation trans-RSN mais, cette fois, le degré d'intervention de l'équipe de communication est bien plus élevé qu'avec une simple capture d'écran, comme le montre l'« éditorialisation » de la parole présidentielle avec l'emploi de différentes polices, de la mise en surbrillance du syntagme nominal the fake news media et de la mention du compte Twitter de Donald Trump. 


\subsection{Les « stories » envisagées comme supports d'interactions}

Les « stories » représentent une fonctionnalité très appréciée sur le RSN Instagram car elles favorisent les interactions entre contributeurs. Les formats de ces stories (qui ont une durée de quelques secondes) incluent tout aussi bien des images enrichies éventuellement de diverses fonctionnalités et filtres que des vidéos de courte durée. Elles sont caractérisées par une durée de vie très limitée car elles sont censées disparaître au bout de 24 heures.

La collecte et la sauvegarde pérenne de stories par les chercheurs représentent ainsi un enjeu important dans l'étude de la matérialité discursive d'un objet intrinsèquement polysémiotique mais aussi dans un but de conservation à visée historique et patrimoniale. En effet, l'archivage d'un jeu de données (correspondant à une saisie instantanée comme une capture d'écran visant à conserver une image ou une capture vidéo permettant d'enregistrer une story) n'est pas proposé par défaut sur la plateforme Instagram. Les données enregistrées pour cette étude se réduisent ainsi à des captures d'écran. Dans le cas de vidéos, ces captures d'écran s'apparentent à des instantanés, les paramètres vocaux d'un discours et les mouvements et actions du contributeur étant, par essence, neutralisés.

La visite d'Emmanuel Macron le 4 octobre 2019 au Sommet de l'élevage (salon européen des professionnels de l'élevage) à Cournon-d'Auvergne a donné lieu à 18 courtes vidéos. La première story (la plus ancienne chronologiquement) est illustrée par une capture d'écran montrant le président lors de son arrivée au salon, une image fixe sur laquelle sont indiqués le fragment textuel «Salon de l'élevage» à valeur de topique et le technomot/technolien « Cournon-d'Auvergne » assurant une fonction de géolocalisation :

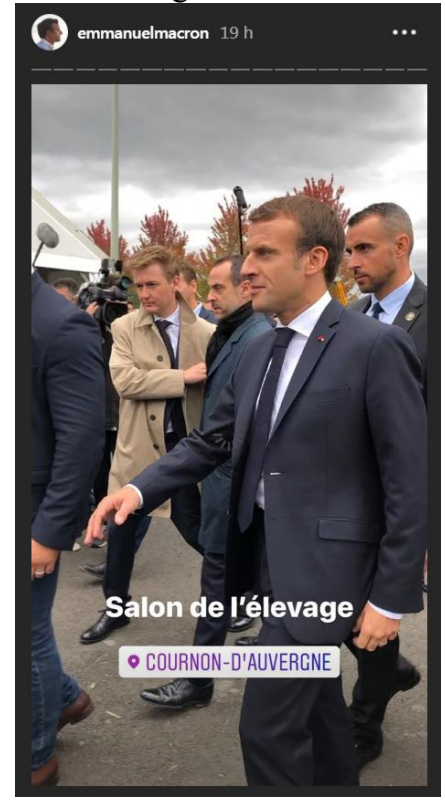

Fig. 8. Story d'Emmanuel Macron : vidéo au Sommet de l'élevage.

Les autres stories présentent des vidéos montrant le président s'entretenant avec des éleveurs sans processus d'éditorialisation (les vidéos sont diffusées sans ajout d'éléments textuels ou de technographismes) : 


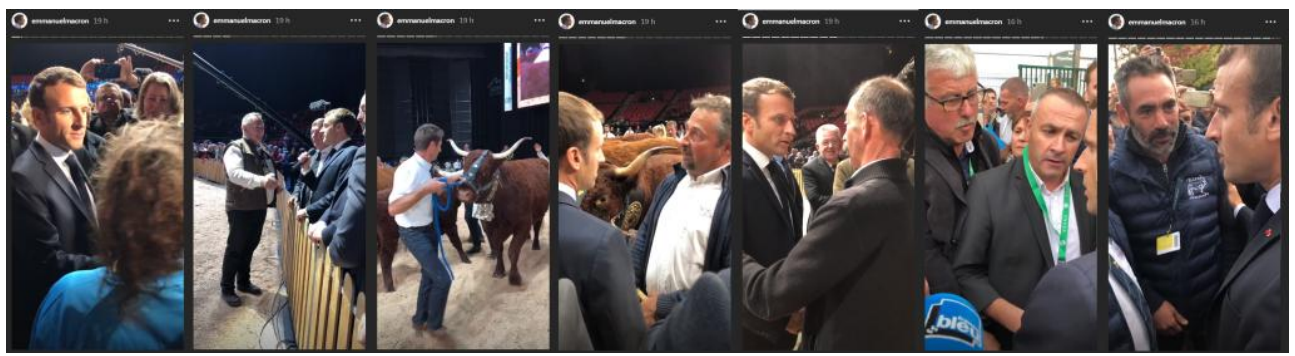

Fig. 9-15. Différentes stories d'Emmanuel Macron au Sommet de l'élevage.

Les stories en tant que publications multimodales interactives peuvent faire l'objet de personnalisations avec l'utilisation des nombreux effets techniques proposés : filtre, tag de localisation, mention d'un autre compte Instagram, hashtag, musique en fond sonore, heure de réalisation du support iconographique, sondage, GIF, questions, compte à rebours, quizz... ainsi qu'un très grand choix d'emojis (ou émoticônes), ajout également de textes dans différentes couleurs et polices. Les diverses personnalisations des stories font qu'il est possible d'envisager un très grand nombre de combinaisons d'actions techniques possibles pour une seule story.

D'une manière plus générale, les stories sont envisagées comme des canaux de diffusion supplémentaires pour partager l'actualité d'un contributeur en lien avec un événement donné (ici, l'inauguration d'un salon). Elles favorisent l'instantanéité, les vidéos " brutes » diffusées pouvant donner l'illusion d'une absence de médiation entre l'événement filmé et les contributeurs qui visionnent ces stories.

De plus, les stories vidéos dont les captures d'écran sont présentées ci-dessus (figures 9 à 15) contenaient des prises de parole du président et de ses interlocuteurs : elles offrent un contenu enrichi par rapport à de simples images fixes. Ces stories se présentent comme de courtes séquences vidéo à l'instar de reportages capturant l'actualité sur le vif.

Les contributeurs sont avertis de la présence de stories non visionnées sur un compte lorsque la photo de profil est entourée d'un disque lumineux orangé. Le gestionnaire du compte peut choisir les paramètres de chaque story : il peut autoriser, lors du visionnage, l'envoi de commentaires (saisie dans la zone «Envoyer un message ») et, le cas échéant, le partage de photos ou de vidéos en réponse à une story, l'expression d'émotions via un clic sur des émoticônes prédéfinies. Il peut également autoriser le visiteur à partager cette story sur un compte tiers. La story d'origine peut alors être enrichie d'éléments ajoutés par le contributeur.

\subsection{Les actions techniques pour citer ou partager une publication ou une story}

Les stories, tout comme les contributions classiques postées sur Instagram, ne sont configurables qu'à partir d'un support multimédia comme un smartphone ou une tablette. Il n'est ainsi pas possible de créer une story à partir d'un navigateur Internet.

Les actions techniques (qui s'appuient sur l'exploitation de la créativité du contributeur) reposent ainsi sur la mise en œuvre de processus haptiques par l'exploitation des propriétés physiques offertes par les écrans tactiles ou encore par des clics sur une souris ou l'appui sur les touches d'un clavier d'ordinateur. Les actions accomplies par le contributeur dans le processus de diffusion et de partage d'une publication ou d'une story consistent en l'activation de technographismes ou de « signes passeurs ${ }^{13}$.

Le processus citationnel mis en œuvre peut consister à reprendre verbatim le contenu plurisémiotique de la publication originelle (en cela, le partage ressemble dans son principe à un « retweet servile ${ }^{14}$, comme on peut le générer sur la plateforme Twitter). 


\section{Conclusion}

Les matérialités des discours numériques circulant sur Instagram sont régies par les propriétés langagières et techniques offertes par la plateforme technologique de ce RSN. Sur tous les RSN dont Instagram, les discours générés dans ces publications numériques sont composites, combinant une composante langagière et une composante technologique.

Une inflexion paradigmatique de l'analyse du discours s'impose pour pouvoir analyser non seulement le contenu textuel mais aussi les contenus plurisémiotiques (emojis, sons, images, vidéos...) qui composent les publications postées sur les RSN. L'incomplétude ontologique de ces RSN s'accompagne de divers processus de délinéarisation et d'hypertextualité.

Les publications et stories que les contributeurs peuvent poster et partager sur Instagram exploitent les diverses fonctionnalités techniques offertes par le RSN et, notamment, l'usage de technosignes qui mettent en avant la dimension réticulaire d'Instagram en favorisant les interactions et les relations entre contributeurs.

L'analyse des publications et stories du corpus de recherche a permis de montrer comment les pratiques citationnelles s'adaptent et se renouvellent en permanence au gré de l'ajout de nouvelles "affordances " ${ }^{15}$ numériques et de la mise en œuvre de processus haptiques qui permettent aux contributeurs d'exploiter ces fonctionnalités technologiques.

Nous avons vu comment des responsables politiques peuvent exploiter les fonctionnalités techniques des RSN dans leur communication institutionnelle. La plurisémioticité des publications numériques complexifie le dispositif énonciatif qui met en avant des citations. La citation transmédiale (et trans-RSN) permet aux leaders politiques une diffusion massive de leurs publications auprès de communautés de contributeurs très diverses pour augmenter le nombre des visites et s'affranchir des distances géographiques et sociales pour afficher une certaine proximité avec leurs concitoyens.

L'offre combinée des deux types de publications offertes par Instagram encourage de nouveaux usages citationnels favorisant la mise en scène des activités (déclarations, meetings, visites...) des responsables politiques. L'effet d'instantanéité mis en avant sur ce RSN contribue pleinement à la création d'une identité numérique et d'un ethos socionumérique pour chaque leader politique, un ethos qui s'affine et évolue au gré de chaque nouvelle publication.

\section{Références bibliographiques}

\section{Corpus numériques}

Comptes Instagram : @emmanuelmacron, @realdonaldtrump

\section{Articles et ouvrages}

Angé, C. (éd.). (2015). Les objets hypertextuels : pratiques et usages hypermédiatiques. Londres: ISTE éditions.

Barats, C, Leblanc, J.-M. et Fiala, P. (2017 [2016]). Approches textométriques du web : corpus et outils. In Barats, C. (éd.), Manuel d'analyse du web, Paris : Armand Colin, 107-127.

Basso Fossali, P., Colas-Blaise, M. et Dondero, M. G. (éd.). (2019). La communication à l'épreuve du geste numérique. Paris : L'Harmattan.

Bigey, M. et Simon, J. (éd.). (2018). Analyse des discours d'escorte de communication sur Twitter : essai de typologie des tactiques d'accroches et de mentions. In Mercier, A. et Pignard-Cheynel, N. (éd.), \#info : commenter et partager l'actualité sur Twitter et Facebook, Paris : Éditions de la Maison des sciences de l'homme, 55-86. 
Burger, M., Thornborrow, J. et Fitzgerald, R. (2017). Introduction. In Burger, M., Thornborrow, J. et Fitzgerald, R. (éd.), Discours des réseaux sociaux; enjeux publics, politiques et médiatiques, Louvain-la-Neuve : De Boeck Supérieur, 9-24.

Chevet, C. et Garmon, I. (2019). Le geste et la parole à l'ère du numérique : de quoi le swipe et la commande vocale sont-ils l'énonciation? Sur les gestualités énonciatives dans les interfaces naturelles. In Basso Fossali, P., Colas-Blaise, M. et Dondero M. G. (éd.), La communication à l'épreuve du geste numérique, Paris : L'Harmattan, 131-144.

Constantin de Chanay, H. et Rosier, L. (2016). Faces extimes sur Facebook: un point de vue «personnel ». Cahiers de praxématique, $n^{\circ} 66$, http://journals.openedition.org/praxematique/4237. Consulté le $1^{\text {er }}$ octobre 2019.

Davallon, J., Després-Lonnet, M., Jeanneret, Y., Le Marec, J. et Souchier, E. (2003). Introduction. In Souchier, E., Jeanneret, Y. et Le Marec, J. (éd.), Lire, écrire, récrire : objets, signes et pratiques des médias informatisés, Paris : Bpi/Centre Pompidou, 19-43.

Develotte, C. et Paveau M.-A. (2017). Pratiques discursives et interactionnelles en contexte numérique. Questionnements linguistiques. Langage et société, n¹60-161, 199-215.

Genette, G. (1982). Palimpsestes. La littérature au second degré. Paris : Seuil.

Grossmann, F. et Rosier L. (2018). Quelques aspects de l'évidentialité hypertextuelle : relations entre discours rapporté et discours d'arrière-plan. In Simon, J. (éd.), Le discours hypertextualisé : espaces énonciatifs mosaïques, Besançon : Presses Universitaires de Franche-Comté, 41-64.

Jackiewicz, A. (2016). Reprises et détournements polémiques sur Twitter. Travaux de linguistique, $n^{\circ} 73,85-105$.

Krieg-Planque, A. (2011). Les «petites phrases »: un objet pour l'analyse des discours politiques et médiatiques. In Krieg-Planque, A. et Ollivier-Yaniv, C. (éd.), Les "petites phrases » en politique, Communication \& Langages, $n^{\circ} 168,23-41$.

Longhi, J. et Weber, J. (éd.). (2018). La communication numérique, du code à l'information. Paris : L'Harmattan.

López Muñoz, J. M. (2017). Tweets politiques et image de twitteur : l'exemple de @fhollande. In Corcuera, J. F., Gaspar, A., Djian, M., Vicente, J. et Bernal, C. (éd.), Les discours politiques : regards croisés, Paris : L’Harmattan, 470-482.

Maingueneau, D. (2004). Hyperénonciateur et « particitation ». Langages $n^{\circ} 156,111-126$.

Nerlich, M. (1990). Qu'est-ce qu'un iconotexte? Réflexions sur le rapport texte-image photographique dans La Femme se découvre d'Evelyne Sinnassamy. In Montandon, A (éd.), Iconotextes, Paris : Ophrys, 255-302.

Paveau, M.-A. (2017). L'analyse du discours numérique : dictionnaire des formes et des pratiques. Paris : Hermann Éditeurs.

Paveau, M.-A. (2016). Des discours et des liens. Hypertextualité, technodiscursivité, écrilecture. Le discours hypertextualisé. SEMEN, n 42, 23-48.

Paveau, M.-A. (2015). En naviguant en écrivant. Réflexions sur les textualités numériques. In Adam, J.-M. (éd.), Faire texte : frontières textuelles et opérations de textualisation, Besançon : Presses Universitaires de Franche-Comté, 337-353.

Petitjean, A.-M., Cordier, A. et Alamargot, D. (2017). Présentation. Le français aujourd'hui, $n^{\circ} 196$, 3-10.

Reboul-Touré, S. (2016). Les tweets : un lieu pour la créativité lexicale ? In Jacquet-Pfau, C. et Sablayrolles, J.-F. (éd.), La fabrique des mots français. Limoges : Lambert-Lucas, 313-326.

Saemmer, A. (2015). Rhétorique du texte numérique. Figures de la lecture, anticipations de pratiques. Villeurbanne : Presses de l'Enssib. 
Simon, J. et Toullec, B. (2018). «Quand les tweets avec images renouvellent le partage d'informations ». In Mercier, A. et Pignard-Cheynel, N. (éd.), \#info : commenter et partager l'actualité sur Twitter et Facebook, Paris: Éditions de la Maison des sciences de l'homme, 131-168.

Sitri, F. et Barats, C. (2017). Constituer un corpus en analyse du discours, un moment crucial. In Née, É. (éd.), Méthodes et outils informatiques pour l'analyse des discours, Rennes: Presses universitaires de Rennes, 41-62.

\footnotetext{
${ }^{1}$ Le corpus constitué pour cette recherche est présenté dans la sous-partie 2.4.

${ }^{2}$ Le terme hypertexte est emprunté à G. Genette (1982:11-12) qui le définit ainsi : «J'entends par là toute relation unissant un texte B (que j'appellerai hypertexte) à un texte antérieur A (que j'appellerai, bien sûr, hypotexte) sur lequel il se greffe d'une manière qui n'est pas celle du commentaire ».

${ }^{3}$ Ce RSN, très populaire aux États-Unis, est utilisé de préférence par les photographes professionnels pour héberger et présenter de manière ordonnée leurs galeries de photos dans des dossiers thématiques.

${ }^{4}$ Les «technomots » sont « définis comme des unités cliquables permettant la circulation par écrilecture d'une source textuelle à une cible textuelle » (Paveau, $2017: 26$ )

${ }^{5}$ Le hashtag permet avant tout «la redocumentarisation d'un thème » (Paveau, $2017: 346$ ) en liant tous les énoncés partageant un sujet commun.

${ }^{6}$ Ces boutons « servent à partager des contenus, à les liker ou à effectuer d'autres opérations [...]. Ce sont des technographismes producteurs de discours, autrement dit des technosignes » (Paveau, $2017: 319$ ).

${ }^{7}$ Il faut noter que la publication d'une « image-texte » est aussi très souvent utilisée sur Twitter pour contourner la limite des 280 signes.

${ }^{8}$ Le terme est emprunté à D. Maingueneau (2004).

${ }^{9}$ A. Krieg-Planque (2011:26) en donne la définition suivante : une « "petite phrase" est un syntagme dénominatif métalinguistique non-savant (et plus précisément : relevant du discours autre approprié), qui désigne un énoncé que certains acteurs sociaux rendent remarquable et qui est présenté comme destiné à la reprise et à la circulation $»$.

${ }^{10}$ Ce hashtag contient une intertextualité forte puisqu'il fait écho au hashtag \#MakeAmericaGreatAgain popularisé par Donald Trump dans sa campagne présidentielle en 2016.

${ }^{11}$ L'écrilecture est intrinsèquement liée à l'hypertextualité qui caractérise les publications des RSN : «l'hyperlien se repère graphiquement (couleur et/ou soulignement) et donne [au lecteur] le choix de continuer sa lecture linéairement ou de cliquer et de se laisser "adresser" à un texte cible : sa lecture est alors une écrilecture puisqu'il écrit, en le lisant, un autre texte que celui qui se présente superficiellement à lui ; le lecteur est un écrilecteur » (Paveau, $2017: 218$ ).

${ }^{12}$ Selon M. Nerlich (1990:268, cité par Simon et Toullec $\left.2018: 137\right)$, l'iconotexte est défini comme « une unité indissoluble de texte(s) et image(s) dans laquelle ni le texte ni l'image n'ont de fonction illustrative ». Ainsi, l'image et le texte ont une importance quasi équivalente, la dimension iconographique et la dimension textuelle occupant toutes les deux une place essentielle dans l'expression sémiotique d'un contenu composite.

${ }^{13}$ Le terme signes passeurs est défini ainsi par J. Davallon et al. (2003:23) : «"les signes passeurs" [...] nous donnent accès aux multiples modalités du texte. Ainsi des "icônes", "boutons", "flèches de navigation" et autres mots "hypertextualisés". Ces signes n'assurent pas seulement une fonction instrumentale permettant de "circuler" dans le texte lui-même, mais présentent également une fonction sémiotique complexe ».

${ }^{14}$ Le terme est emprunté à M. Bigey et J. Simon (2018:57).

15 Selon M. Burger et al. (2017:12), les «affordances» sont les «caractéristiques technologiques des plateformes » numériques.
} 